\title{
Patients with insulin-dependent diabetes or coronary heart disease following rehabilitation express serum fractalkine levels similar to those in healthy control subjects
}

This article was published in the following Dove Press journal:

Vascular Health and Risk Management

8 October 2009

Number of times this article has been viewed

\section{Lars Maegdefessel ${ }^{1,3}$ \\ Axel Schlitt' \\ Susanna Pippig' \\ Bernhard Schwaab ${ }^{2}$ \\ Kerstin Fingscheidt ${ }^{2}$ \\ Uwe Raaz' \\ Michael Buerke' \\ Harald Loppnow'}

'Universitätsklinik und Poliklinik für Innere Medizin III, Martin-Luther-

Universität Halle-Wittenberg, Halle

(Saale), Germany; ${ }^{2}$ Klinik Höhenried,

Bernried, Bayern, Germany;

${ }^{3}$ Department of Cardiovascular

Medicine, Stanford University School

of Medicine, Stanford, CA, USA
Correspondence: Lars Maegdefessel Stanford University School of Medicine, Division of Cardiovascular Medicine, 300 Pasteur Drive, Stanford, CA 94305-5406, USA

Tel + I 6507245075

Fax + I 650725 I599

Email maegdefessel@stanford.edu
Abstract: The chemokine and adhesion molecule fractalkine and its receptor $\mathrm{CX}_{3} \mathrm{CR} 1$ have emerged as interesting regulators in inflammation and related atherosclerosis. The pro-inflammatory status may be counteracted by appropriate treatment, such as in rehabilitation. We compared serum fractalkine concentrations of 46 patients with coronary heart disease (CHD) and 47 insulin-dependent diabetic patients (IDDM) following rehabilitation with those of 50 control subjects. Following rehabilitation serum fractalkine levels $(477 \pm 225 \mathrm{pg} / \mathrm{mL})$ in CHD patients were similar to those in control subjects $(572 \pm 205 \mathrm{pg} / \mathrm{mL} ; P=0.303)$, whereas fractalkine levels were lower in IDDM patients $(430 \pm 256 \mathrm{pg} / \mathrm{mL} ; P=0.042)$. No significant difference between CHD and IDDM patients was present $(P=0.319)$. Postprandial hyperlipemia may influence inflammation; thus, we investigated fractalkine levels four and eight hours after inducing postprandial hyperlipemia. However, we did not find any significant alterations in CHD and diabetic patients, whereas the fractalkine levels in controls were reduced. In vitro, lipofundin used as a hyperlipemic stimulus was added to vessel wall cells and reduced fractalkine levels. Low fractalkine levels in patients attending rehabilitation indicate a beneficial effect of the rehabilitation procedure on innate inflammatory pathways, such as the chemokine and adhesion molecule fractalkine.

Keywords: inflammation, cytokines, atherosclerosis, fractalkine, coronary heart disease, diabetes, rehabilitation

\section{Introduction}

Atherosclerosis is a multifactorial disease of the blood vessel wall that is strongly influenced by inflammatory pathways, ${ }^{1,2}$ such as adhesion of leukocytes to the endothelium, and other inflammatory or innate mechanisms. ${ }^{3,4}$ During atherogenesis, monocytes accumulate in the neointima and subsequently transform into tissue macrophages/foam cells. In addition, other leukocytes, such as mast cells ${ }^{5}$ or T-cells, ${ }^{6}$ are also found in atherosclerotic lesions. The invasion of the vessel wall starts when blood cells adhere to the endothelium. However, the mechanisms underlying the initial activation have still not been completely defined. The leukocyte-endothelial interaction involves rolling, firm adhesion, and migration into the neointima, which is mediated by selectins and other adhesion molecules ${ }^{7}$ as well as by chemokines. ${ }^{8}$ Chemokines attract and direct cells along a chemotactic gradient. Furthermore, they may activate other atherosclerosis-relevant functions of vessel wall cells or leukocytes, for example, by activating adhesion molecules on the endothelium. ${ }^{8,9}$ 
The recently discovered chemokine fractalkine ${ }^{10}$ and its receptor $\mathrm{CX}_{3} \mathrm{CR} 1$ have emerged as interesting intermediaries in atherosclerosis. Fractalkine is a multidomain protein expressed on the surface of a variety of cells, including activated endothelial and smooth muscle cells. ${ }^{11-15}$ Fractalkine exhibits features which have not been described for other members of the chemokine family, including its exceptionally large size of $95 \mathrm{kDa}$ and the presence of a transmembrane anchor. Furthermore, a soluble form can be released from its membrane-associated form by extracellular cleavage. ${ }^{16}$ $\mathrm{CX}_{3} \mathrm{CR} 1$ is a seven-transmembrane-spanning fractalkine receptor, mediating its signal by G-proteins, as most cytokines do. This receptor mediates the firm adhesion to fractalkine even in the absence of G-protein activation ${ }^{17}$ and thus plays a role as an adhesion molecule in addition to functioning as a chemokine. In this way, membrane-bound fractalkine contributes to the adhesion of $\mathrm{CX}_{3} \mathrm{CR} 1$-expressing leukocytes. On the other hand, soluble fractalkine, after enzymatic cleavage and release, has potent chemotactic activity for monocytes and lymphocytes but, like CC chemokines, not for granulocytes. ${ }^{18}$

Cytokines, including chemokines, and other innate compounds are strongly involved in the inflammatory pathways contributing to atherosclerosis..$^{4,7}$ Thus, evidence has been provided that fractalkine plays a role in various cardiovascular diseases. For example, increased fractalkine levels have been reported in patients after cardiac allograft rejection. In a murine model, allograft survival was prolonged from $7 \pm 1$ days to $49 \pm 30$ days $(P \leq 0.0008)$ by administering fractalkine antibodies. ${ }^{19}$ These studies identified a critical role for fractalkine in the pathogenesis of acute rejection and suggest that fractalkine inhibition might be a potential therapeutic target. Fractalkine levels have only been rarely determined in patients; however, it has been shown that Alzheimer patients express enhanced fractalkine levels, correlating with the Mini-Mental State Examination (MMSE) score. ${ }^{20}$ Another study also reported that fractalkine levels are enhanced in Wegener's granulomatosis. ${ }^{21}$ In patients with coronary artery disease fractalkine plasma levels were enhanced and then reduced after statin therapy. ${ }^{22}$ When $\mathrm{CX}_{3} \mathrm{CR} 1$ genotypes were analyzed in patients with acute coronary syndromes and healthy controls, $\mathrm{CX}_{3}$ CR1-I249 heterozygosity was associated with a markedly reduced risk of acute coronary events, independent of established coronary risk factors such as smoking or diabetes. ${ }^{23,24}$

Both the risk and the rate of development of atherosclerosis are increased in diabetic patients, but the underlying mechanisms are still not completely understood.
Epidemiologic and pathophysiologic evidence suggests that lipoproteins, platelets, or soluble clotting factors represent contributing factors. Furthermore, both balance of the prostaglandin-metabolites prostacycline and thromboxane and blood pressure regulation are disturbed. On the other hand, the metabolism and proliferation of arterial smooth muscle cells are modified. ${ }^{25}$ Many of these alterations accompany hyperinsulinemia and may account for the recent evidence that hyperinsulinemia is a risk factor for atherosclerosis. Cytokines and chemokines may contribute to regulate atherosclerosis-related pathways in diabetes or its associated diseases. ${ }^{26,27}$ However, little is known about the expression of fractalkine and its receptor in diabetes, although the mRNA of fractalkine and its receptor were upregulated in diabetic rats in the early stages of diabetic nephropathy. ${ }^{28}$

To the best of our knowledge, fractalkine levels have not yet been analyzed in patients with insulin-dependent diabetes following rehabilitation. An optimized medical treatment consisting of medication such as angiotensinconverting enzyme (ACE) inhibitors, angiotensin-receptor blockers (ARBs), and statins plays an important role during rehabilitation. Some of the aforementioned drugs may also exert anti-inflammatory properties, which could contribute to their beneficial effects. Indeed, fractalkine levels were reduced in patients with coronary artery disease after a six-week statin treatment. ${ }^{22}$ Thus, rehabilitation may normalize levels of fractalkine.

In diabetic patients postprandial hyperlipemia may represent a potential risk factor for atherosclerosis. ${ }^{29}$ Postprandial hyperlipemia can induce an inflammatory state by activating granulocytes,${ }^{30}$ leukocytes, ${ }^{31}$ or endothelial cells. ${ }^{32}$ Triglyceride-rich lipids (TRL) may even penetrate the arterial wall and activate vascular smooth muscle cell genes differently, depending on the composition (ie, butter, vegetable oil/fish oil, or olive oil) of the lipid. ${ }^{33}$ Furthermore, chylomicron remnants containing TRLs have been shown to potently activate smooth muscle cell MCP-1 production. ${ }^{34}$ Postprandial hyperlipemia may promote inflammation in patients and oppose therapy. However, it is not clear whether therapy during rehabilitation may prevent postprandial hyperlipemia from inducing inflammation.

Thus, in the present study we investigated the expression of serum fractalkine in patients with coronary heart disease (CHD) and patients with insulin-dependent diabetes following optimized medical treatment after a period of rehabilitation. Subsequently, we analyzed the effect of postprandial hyperlipemia. We found that fractalkine levels in CHD and 
diabetic patients after rehabilitation were comparable to those in control subjects and that the postprandial fractalkine levels were not significantly increased. However, postprandial fractalkine levels in control subjects were reduced, a finding paralleled by in vitro cell culture data. These data indicate that the anti-inflammatory effects of the rehabilitation medication played an important role in the study patients.

\section{Material and methods}

\section{Study population}

We determined the serum concentrations of fractalkine in 46 nondiabetic patients with angiographically proven CHD, 47 insulin-dependent diabetic patients (IDDM), and 50 healthy controls. The patients were recruited in the "Curschmann Klinik für Rehabilitation" (Timmendorfer Strand/Germany). The control subjects were volunteers (mostly working at the Curschmann Clinic) with no prove of CHD or diabetes mellitus. The study was approved by the ethics committee of the University of Lübeck, Schleswig Holstein, Germany. All patients gave written informed consent.

\section{Blood sampling and clinical laboratory parameters}

Blood samples were taken from all subjects under standardized conditions after an overnight period of fasting (time-zz [compared below]). The next blood samples were taken four and eight hours later. All blood samples were immediately centrifuged at 4,000 rpm for 10 minutes, supernatants were extracted and divided into aliquots. All aliquots were stored at $-80{ }^{\circ} \mathrm{C}$ until analysis. The fractalkine levels in the samples were measured by using a sandwich ELISA (enzyme-linked immunosorbent assay; DuoSet, R\&D Systems, Wiesbaden, Germany) according to the manufacturer's recommendations. Besides fractalkine, atherosclerosis-relevant serum markers of inflammation (C-reactive protein [CRP]) and lipid metabolism (low-density lipoprotein cholesterol [LDL-C]; high-density lipoprotein cholesterol [HDL-C]; total cholesterol [TC]; and triglycerides [TG]) were measured. The lipid markers were routinely determined by standard clinical laboratory analysis.

\section{Induction of postprandial hyperlipemia}

Postprandial hyperlipemia was induced by eating a high calorie fatty meal. Briefly, the meal contained a total of $1,265 \mathrm{kcal} / \mathrm{m}^{2}$ body surface area. It was composed of
$105 \mathrm{~g}$ fat (consisting of approximately $52 \mathrm{~g}$ saturated fat, $53 \mathrm{~g}$ unsaturated fat, and $300 \mathrm{mg}$ cholesterol), $48 \mathrm{~g}$ carbohydrates, and 32 g protein. Probands were instructed to eat the meal within 15 minutes and not to eat any other food until the subsequent blood samples were taken. Before the meal (time-point 0) the diabetic patients received a dose of insulin subcutaneously, which was individually adapted to the meal described above. Glucose levels were measured every two hours until the end of the study period in order to prevent severe hypo- or hyperglycemia. All glucose levels were in an acceptable range; thus, no glucose or further insulin administration was required until the end of the eight-hour study period.

\section{Isolation and culture of human vascular vessel wall cells}

Human vascular smooth muscle cells were isolated from unused portions of saphenous veins obtained following bypass surgery, as described previously. ${ }^{35,36}$ Use of the specimens that would otherwise have been discarded was approved by the local ethics committee. Dulbecco's modified Eagle's medium (DMEM; 1 g/L D-glucose) containing $10 \%$ fetal calf serum (FCS), 1\% antibiotics, and 1\% L-glutamine (DMEM medium) was used for cell culture. Upon confluency the cells were subcultured after treatment with trypsin/EDTA $(0.05 \% 0.02 \%, 10$ minutes, $37{ }^{\circ} \mathrm{C}$, Biochrom, Berlin, Germany). We used smooth muscle cells in passages 4 to 7 . Stimulation experiments were performed in 24-well culture plates as specified in Figure 3. Lipofundin LCT-10 solution was obtained from Braun (Melsungen, Germany).

\section{Statistical analysis}

Statistical analysis was performed with the Statistical Package for the Social Sciences (SPSS Inc, Chicago, IL, USA). Continuous variables were compared by ANOVA. Chi-squared test was used for categorical variables. Correlation analysis was conducted according to the Pearson method.

\section{Results \\ Baseline characteristics}

The demographic data for the study populations are presented in Table 1. Compared with CHD patients and control subjects, the IDDM patients were older. The BMI was not significantly different, and the distribution of sexes was similar among the three groups. More smokers were found 
Table I Characteristics of the investigated study populations ${ }^{\mathrm{a}}$

\begin{tabular}{llllll}
\hline Parameters $^{\mathrm{b}}$ & Unit & Control & IDDM & CHD & $\boldsymbol{P}_{\text {value }}$ \\
\hline Age & years & $52.7 \pm \mathrm{II}$ & $64.0 \pm 9$ & $55.5 \pm 9$ & $<0.00 \mathrm{I}$ \\
BMI & & $26.6 \pm 5$ & $28.2 \pm 5$ & $26.8 \pm 4$ & 0.136 \\
Male & $\%$ & 82 & 78 & 84 & 0.735 \\
Active smokers & $\%$ & 34 & 10 & 2 & $<0.00 \mathrm{I}$ \\
Hypertension & $\%$ & 20 & 90 & 98 & $<0.00 \mathrm{I}$ \\
CHD-FH & $\%$ & 6 & 18 & 42 & $<0.001$ \\
Statins & $\%$ & 4 & 96 & 96 & $<0.00 \mathrm{I}$ \\
$\beta$-Blockers & $\%$ & 10 & 68 & 98 & $<0.001$ \\
ACE inhibitors & $\%$ & 8 & 62 & 56 & $<0.001$ \\
ARB & $\%$ & 2 & 14 & 16 & $<0.001$ \\
Diuretics & $\%$ & 10 & 42 & 20 & $<0.001$ \\
\hline
\end{tabular}

Notes: apatients with insulin-dependent diabetes (IDDM; $n=47$ ) or coronary heart disease (CHD; $n=46$ ) were compared to healthy controls $(n=50)$. Values are presented as mean \pm standard deviation; ${ }^{\mathrm{b}}$ The $P$-value for comparison between all three groups was calculated by ANOVA. The values of the categorical variables are compared by chi-squared test.

Abbreviations: $A C E$, angiotensin-converting enzyme; $A R B$, angiotensin receptor blocker; ANOVA, analysis of variance; BMI, body mass index; $\mathrm{CHD}-\mathrm{FH}$, family history of coronary heart disease.

among the healthy control subjects than in the two patient groups. On the other hand, more subjects with hypertension or a family history of CHD were present in the patient groups. As anticipated, medical treatment with statins, $\beta$-blockers, ACE inhibitors, ARBs, and diuretics was more prevalent in the patient groups. Except for diuretics, medication was similar in the tested patient groups. Healthy controls did not suffer from any obvious atherosclerotic, metabolic, or inflammatory disease.

\section{The fractalkine levels in diabetic patients are lower than in healthy control subjects}

Fractalkine levels have not been measured yet in diabetic patients following rehabilitation. Thus, we analyzed the expression of fractalkine in the IDDM and CHD patients who received optimal medical treatment and underwent rehabilitation for four weeks. The fractalkine levels of the patients were compared to those of the control subjects. A comparison of the fractalkine levels in CHD and IDDM patients after rehabilitation and healthy controls (timepoint 0 ) is shown in Figure 1. At this time-point fractalkine levels were slightly but not statistically significantly lower in CHD patients $(477 \pm 225 \mathrm{pg} / \mathrm{ml})$ than in control subjects $(527 \pm 205 \mathrm{pg} / \mathrm{ml} ; P=0.303)$. In contrast, they were lower in IDDM patients than in the controls $(430 \pm 256 \mathrm{pg} / \mathrm{ml}$;
$P=0.042$ ), whereas they were not significantly different from fractalkine levels in CHD patients (IDDM vs CHD; $P=0.319)$. Thus, fractalkine levels in both patient groups were below or equal to control levels.

\section{Postprandial hyperlipemia does not reduce fractalkine levels in IDDM and CHD patients}

Hyperlipemia can activate inflammatory pathways. However, it was unclear whether postprandial hyperlipemia affects inflammatory parameters in rehabilitation patients as compared to control subjects. Thus, after the first blood sample was taken (time-point 0), a defined postprandial hyperlipemia was induced in the three investigated groups. Four and eight hours after the first blood sampling and eating the defined meal the next samples were taken (time-point 4; time-point 8). At time-point 4 the fractalkine levels were higher in diabetic patients $(548 \pm 300 \mathrm{pg} / \mathrm{mL})$ than in CHD patients (437 $\pm 228 \mathrm{pg} / \mathrm{mL} ; P=0.033)$; however, they were not different from the control levels $(474 \pm 203 \mathrm{pg} / \mathrm{mL}$; Figure 2). At time-point 8 the fractalkine levels were $432 \pm 212 \mathrm{pg} / \mathrm{mL}$ in the CHD group, $454 \pm 262 \mathrm{pg} / \mathrm{mL}$ in the IDDM group, and $393 \pm 166 \mathrm{pg} / \mathrm{ml}$ in the control group. These values were not significantly different from each other. Comparing each group at the different time-points by ANOVA revealed that only the differences in the values for healthy control subjects were statistically significant $(P=0.004)$. Post hoc analysis showed that the fractalkine

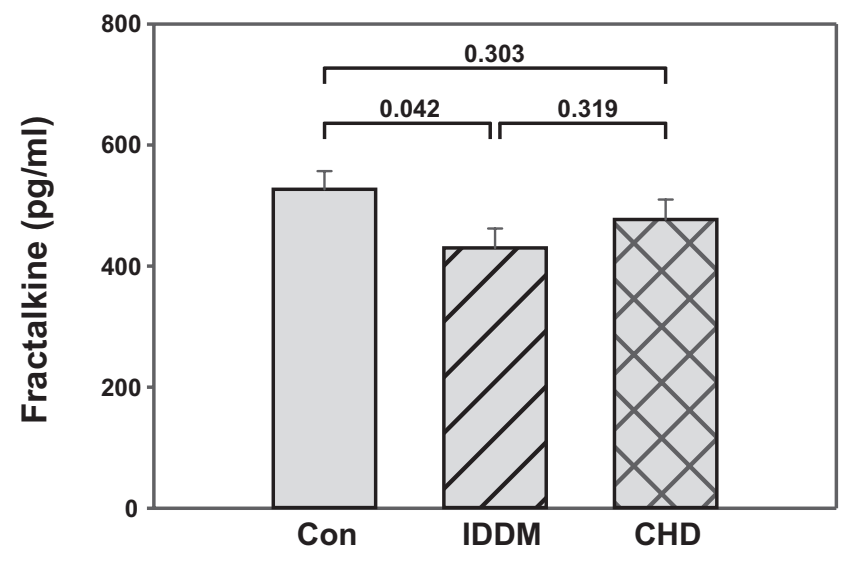

Figure I Fraktalkine levels in patients following rehabilitaiton are not higher than fractalkine levels in healthy control subjects. Blood samples of diabetic patients (IDDM; $n=47)$ and coronary heart disease patients (CHD; $n=46)$ following rehabilitation and blood samples of apparently healthy volunteers (Con; $n=50)$ were measured by fractalkine ELISA. Mean and standard error of the mean are presented. Significances are given above the columns.

Abbreviations: CHD, coronary heart disease; Con, control; ELISA, enzyme-linked immunosorbent assay; IDDM, insulin-dependent diabetic patients. 


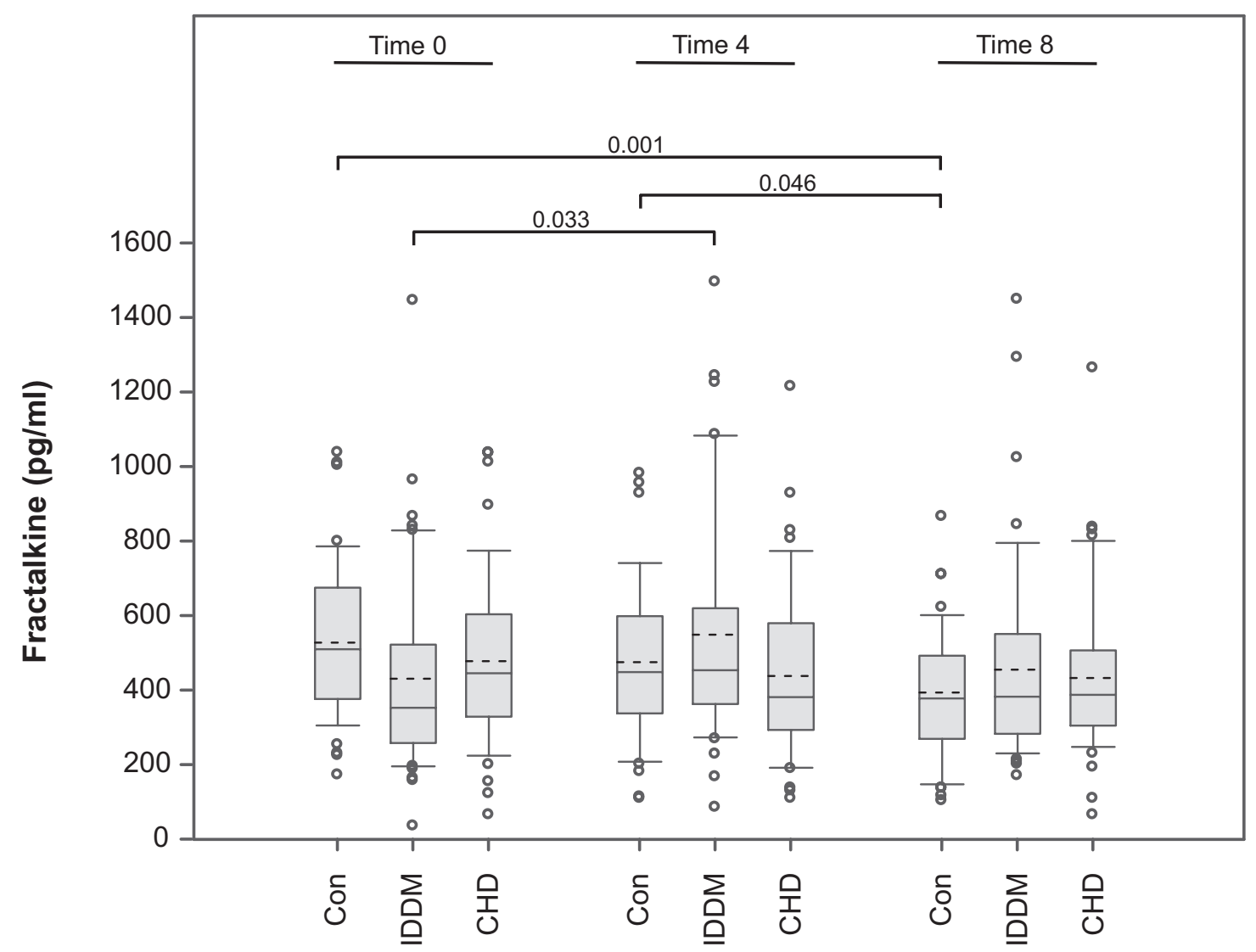

Figure 2 Induction of postprandial hyperlipemia reduces fractalkine levels in healthy control subjects, but not in patients following rehabilitation. Blood samples of IDDM and CHD patients following rehabilitation and blood samples of healthy subjects (Con) were collected at time-point 0 . Immediately thereafter a postprandial hyperlipemia was induced by a defined meal of $\mathrm{I}, 285 \mathrm{kcal} / \mathrm{m}^{2}$ body suface $(105 \mathrm{~g}$ fat $+300 \mathrm{mg}$ cholesterol $+48 \mathrm{~g}$ carbohydrates $+32 \mathrm{~g}$ protein. At two time-points later (four and eight hours, respectively) blood samples were taken and measured by fractalkine ELISA. Data are presented as a box blot (SigmaPlot). The whiskers and box lines indicate the 10th, 25th, median, 75th, and 90th percentiles. The broken line indicates the mean. Extra symbols indicate outliers. Analysis by one-way ANOVA showed a significant difference between the fractalkine levels at the time-points of the control subjects $(P=0.004)$, whereas levels for the diabetic $(P=0.080)$ and the CAD $(P=0.574)$ patients were not significantly different at the given time-points. Post-hoc analysis at the $0.0 \mathrm{I}$ level showed the same result; however, diabetic patients at the time-point 0 and 4 were different at the 0.05 level $(P=0.033)$.

Abbreviations: ANOVA, analysis of variance; CAD, coronary artery disease; CHD, coronary heart disease; Con, control; ELISA, enzyme-linked immunosorbent assay; IDDM, insulin-dependent diabetic patients.

levels at time-point 8 were different from those at time-point 4 $(P=0.046)$ and time-point $0(P=0.001)$, whereas at timepoints 0 and 4 they were not significantly different. In contrast to control subjects, ANOVA analysis of IDDM and CHD patients did not reveal significant differences between the three time-points $(P=0.080$ and $P=0.574$, for diabetes and CHD, respectively). Post hoc analysis showed the same result at the 0.01 level. However, the IDDM time-points 0 and 4 were different at the 0.05 level $(P=0.033)$. Taken together, the data indicate that the fractalkine levels were only reduced in control subjects, whereas in CHD the levels remained low and in IDDM patients the levels fluctuated, although the differences were not significant according to ANOVA. In order to investigate the influence of hyperlipemia in vitro we incubated vascular smooth muscle cells, which represent a potential source of fractalkine in atherogenesis, with several lipofundin concentrations. We observed a dose-dependent reduction in fractalkine production (Figure 3 ).

\section{The fractalkine levels in the tested subjects do not correlate with CRP and lipids}

The analysis of inflammatory and lipid markers at time-point 0 is presented in Table 2. The CRP levels were higher in the diabetic patients $(10 \pm 16 \mathrm{mg} / \mathrm{L})$ than in CHD patients $(6 \pm 7 \mathrm{mg} / \mathrm{L} ; P=0.025)$ and controls $(2 \pm 3 \mathrm{mg} / \mathrm{L}$; $P \leq 0.001)$. All lipid parameters were highest in the healthy control subjects. The differences between controls and IDDM patients were less pronounced than the differences between controls and CHD patients. These data indicate that the lipid-lowering aspect of the IDDM and CHD medication worked properly. The correlation analysis revealed that there was no significant correlation between fractalkine serum 


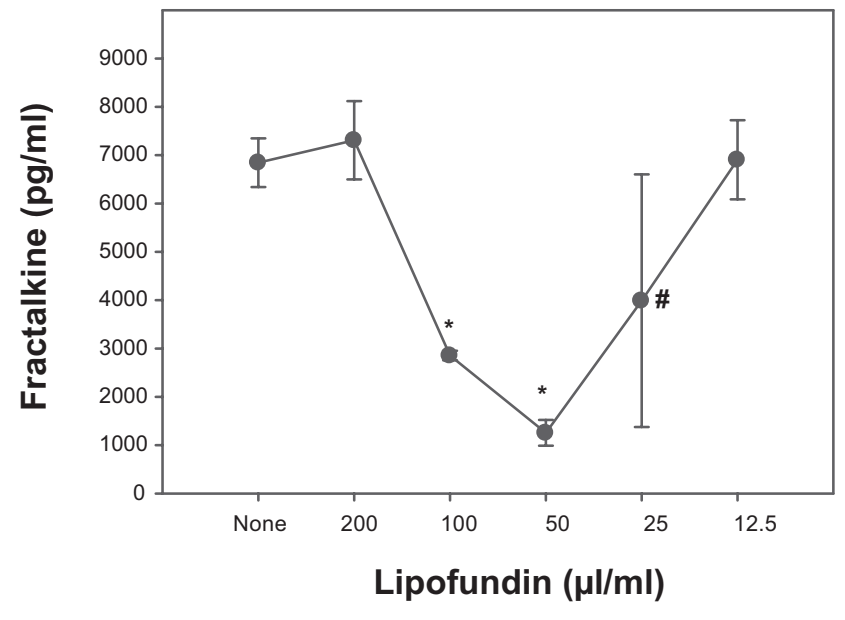

Figure 3 Reduction in fractalkine levels in lipofundin-treated cultures of vessel wall cells. Human vascular smooth muscle cells $\left(20,000 \mathrm{SMC} / \mathrm{cm}^{2}\right)$ were cultured in 24 -well plates. After 24 hours the medium was changed and fractalkine production induced by costimulation with interferon- $\gamma$ and tumor necrosis factor- $\alpha(200 \mathrm{U} / \mathrm{ml}$, $20 \mathrm{ng} / \mathrm{ml}$, respectively). Parallel cultures were incubated in the presence of various concentrations of lipofundin. After 24 hours, the supernatants were harvested and measured by fractalkine ELISA. Two experiments provided similar results.

Abbreviations: ELISA, enzyme-linked immunosorbent assay; SMC, smooth muscle cells.

levels, CRP, the lipid markers, age, or body mass index (BMI). BMI did not correlate with cholesterol, triglycerides, or LDL either. On the other hand, and as expected, BMI inversely correlated with HDL-C $(-0.264 ; P \leq 0.001)$; the high CRP levels inversely correlated with high HDL levels $(-0.242 ; P \leq 0.001)$; and cholesterol levels correlated well with triglycerides $(0.463 ; P<0.001)$ and LDL-C $(0.847$; $P \leq 0.001)$.

\section{Fractalkine in coronary artery bypass graft surgery and multivessel disease}

Among the CHD patients the subgroup of patients who had currently received coronary artery bypass graft $(\mathrm{CABG})$ surgery were analyzed because these patients express the highest pro-inflammatory state prior to rehabilitation.

Table 2 Classical markers of inflammation and lipid metabolism ${ }^{a}$

\begin{tabular}{llllll}
\hline Parameter & Unit & Control & IDDM & CHD & P value $^{\mathrm{b}}$ \\
\hline CRP & $\mathrm{mg} / \mathrm{L}$ & $2 \pm 3$ & $10 \pm 16$ & $6 \pm 7$ & 0.001 \\
HDL-C & $\mathrm{mg} / \mathrm{dL}$ & $45 \pm 12$ & $39 \pm 22$ & $35 \pm 7$ & 0.009 \\
LDL-C & $\mathrm{mg} / \mathrm{dL}$ & $127 \pm 35$ & $79 \pm 38$ & $68 \pm 21$ & 0.000 \\
TC & $\mathrm{mg} / \mathrm{dL}$ & $205 \pm 35$ & $151 \pm 50$ & $124 \pm 27$ & 0.000 \\
Triglycerides & $\mathrm{mg} / \mathrm{dL}$ & $160 \pm 113$ & $145 \pm 13$ & $101 \pm 45$ & 0.013 \\
\hline
\end{tabular}

Notes: aPatients with insulin-dependent diabetes (IDDM; $n=47$ ) or coronary heart disease $(C H D ; n=46)$ were compared to healthy controls $(n=50)$ at timepoint 0 . Values are presented as mean \pm standard deviation; ${ }^{\text {TThe }} p$-value for comparison between all three groups was calculated by ANOVA.

Abbreviations: CRP, C-reactive protein; HDL-C, high-density lipoprotein cholesterol; LDL-C, low-density lipoprotein cholesterol; TC, total cholesterol.
However, in the 14 patients of the CHD group who had received a $\mathrm{CABG}$ within two weeks before attending the "Curschmann Klinik für Rehabilitation" ( $533 \pm 288 \mathrm{pg} / \mathrm{mL})$, the fractalkine serum levels were not significantly different from those in the CHD patients not receiving a bypass (459 $\pm 203 \mathrm{pg} / \mathrm{mL} ; P=0.349)$. In addition, a CHD subgroup of patients suffering from multivessel disease with either two $(496 \pm 237 \mathrm{pg} / \mathrm{mL})$ or three $(429 \pm 215 \mathrm{pg} / \mathrm{mL})$ stenosed coronary arteries did not express significantly increased fractalkine levels after rehabilitation, as compared to patients with only one $(507 \pm 231 \mathrm{pg} / \mathrm{mL})$ occluded coronary artery $(P=0.575)$.

\section{Discussion}

Innate inflammatory pathways are important in atherogenesis. Chemokines and adhesion molecules contribute to this process. Disease-associated, enhanced levels of adhesion molecules, cytokines, or chemokines may be reduced after optimal medical treatment during rehabilitation. Thus, we compared the serum levels of fractalkine in patients with CHD or IDDM following rehabilitation with those in healthy control subjects. Furthermore, we investigated the relation between fractalkine and classical risk factors for atherosclerosis in patients with CHD and IDDM in a state of postprandial hyperlipemia. The data show that the fractalkine levels of the patients after rehabilitation were at the control level or lower. Postprandial hyperlipemia reduced the fractalkine levels in control subjects and in vessel wall cells in vitro, whereas postprandial hyperlipemia did not result in significantly different fractalkine levels in diabetic and CHF patients according to ANOVA. These data indicate that the fractalkine levels were similar to control values and that acute hyperlipemia did not alter fractalkine levels in patients, whereas it did so in controls.

Previous studies reported that soluble fractalkine was increased in CHD patients as compared to controls, and that membrane-bound fractalkine was increased in human carotid arteries and in animal models. ${ }^{37,38}$ In another study serum fractalkine levels were determined: ${ }^{22}$ These data showed that patients with unstable angina $(1,199 \pm 112 \mathrm{pg} / \mathrm{mL})$ had enhanced fractalkine levels as compared with control levels $(423 \pm 25 \mathrm{pg} / \mathrm{mL})$ and that six-month atorvastatin therapy, but not simvastatin therapy, greatly reduced the fractalkine levels in the investigated patients $(587 \pm 89 \mathrm{pg} / \mathrm{mL})$. These results are in line with the data shown in the present manuscript, which are in the range of those for the controls or statin-treated patients. Fractalkine has been demonstrated to be involved in diabetic nephropathy in murine models. Upregulated fractalkine and 
$\mathrm{CX}_{3} \mathrm{CR} 1$ levels were present in the early stage of diabetic kidney disease in rats. ${ }^{28}$ The aforementioned data suggest that fractalkine and $\mathrm{CX}_{3} \mathrm{CR} 1$ have an important role in the progression of diabetic nephropathy, functioning as an arrest chemokine in monocyte/macrophage adhesion before migration into the kidney. Blockade of the renin-angiotensin-aldosterone system and treatment with statins currently represent the only clinical strategies for treating the inflammatory process in diabetic nephropathy. Newer strategies include chemokine receptor antagonists and even immunosuppressive therapy, but these are still in the experimental stage. In contrast to the CHD patients, no data exist about the fractalkine levels in untreated diabetic patients. We are currently addressing this question. However, the cited data indicate that fractalkine levels are enhanced in CHD patients. The low fractalkine levels in the same patient group in the present study suggest an analogous reducing effect caused by 1) the rehabilitation process, and/or 2) alleviating the acute status of the disease.

Serum fractalkine levels in CHD and IDDM patients following rehabilitation did not differ from those in healthy control subjects. This may partly be explained by the optimal medical and lifestyle treatment that patients benefit from during rehabilitation. Thus, blood glucose, heart rate, and blood pressure are continuously monitored and promptly corrected. Furthermore, patients profit from physical activity and controlled food intake, ie, lifestyle improvement. ${ }^{39,40}$

On the other hand, patients with CHD and IDDM in rehabilitation are no longer in an acute phase of the disease. The baseline characteristics of our study population indicate that medical treatment in the two patient groups was more prevalent than in control subjects. The majority (96\%) of the CHD and IDDM patients were on statin therapy compared to only $4 \%$ in controls. In addition to their lipid-lowering activity, statins may also have anti-inflammatory effects. During the constrictive remodeling status, statins have the ability to stabilize the atherosclerotic plaque. ${ }^{41}$ In addition, their anti-inflammatory effect was measured by their ability to lower CRP levels. ${ }^{42,43}$ The lipid and CRP data in the latter publications are in line with those for the control population presented here. The lipid data in the present study patients also indicate that the treatment had a similarly beneficial effect. However, the CRP levels did not reach the control values, which might be explained by the type and dose of statins used in the current study. This may have influenced the expression of the inflammatory parameters. ${ }^{22,41}$ Other therapeutic agents in atherosclerotic diseases include ACE inhibitors and ARBs. Both agents can reduce the expression of angiotensin I and II receptors on endothelial cells and monocytes. Thereby, they may reduce various inflammatory processes. ${ }^{42,43} \mathrm{ACE}$ inhibitors and $\mathrm{ARB}$ therapy was used in $72 \%$ of the CHD and IDDM patients, compared to only $10 \%$ among the healthy controls. We hypothesize that the low fractalkine levels in the two patient groups may be caused by the combined ACE inhibitor, ARB, and statin treatment.

An approximate measure of treatment efficacy is shown by the differing responses to postprandial hyperlipemia found in the present experiments. Postprandial hyperlipemia significantly reduced fractalkine levels in the controls, whereas it had no effect in the patients. We assume that this lack of response was caused by the anti-inflammatory capacity of the drugs being used. On the other hand, the lower (systemic) fractalkine levels may reflect reduced shedding, which in turn may indicate enhanced vascular inflammation. However, this suggestion remains somewhat speculative since no corresponding data have been published. However, we found that lipid treatment (lipofundin) reduced fractalkine release in vascular smooth muscle cells in a dose-dependent fashion in in vitro experiments. The reduction in the fractalkine levels in the controls also indicates that the selected measurement time-points were adequate. Other reports used similar timepoints for postprandial measurements. ${ }^{30,32}$ On the other hand, we found that cytokines could already be detected in bypass patients after three hours. ${ }^{44}$ The enhanced fractalkine level at the four-hour time-point may reflect activation of the inflammatory genes by insulin. ${ }^{45,46}$ However, insulin is also known to have anti-inflammatory effects.

Similarly to other studies we were not able to show any significant correlation between fractalkine levels and other classical risk factors for atherosclerosis, such as lipid metabolism (HDL-C, LDL-C, TC, and TG), age, BMI, or diabetes. ${ }^{22,23}$ In our study, fractalkine serum levels were not significantly elevated in patients who received CABG surgery within two weeks before attending rehabilitation. This indicates that the acute inflammatory phase in these patients has already subsided. Furthermore, fractalkine levels in patients with two or three occluded coronary arteries were not higher than those in patients with only one stenosed coronary artery. These data suggest that the differences in the inflammatory situations between controls and CHD patients, ${ }^{22}$ or as suspected here, between controls and diabetic patients are leveled by rehabilitation. Serum fractalkine levels are increased in patients with CHD during the acute inflammatory state of the disease, such as acute myocardial infarction or acute heart failure. ${ }^{22,47}$ After the acute phase of a cardiovascular inflammatory disease optimized medical treatment, such as that given during rehabilitation, may 
decrease the inflammatory status, as shown by the similar serum fractalkine levels in patients and healthy controls in this study.

\section{Conclusion}

Our results demonstrate the relevance of optimized medical treatment, such as that given during rehabilitation, for patients with CHD and IDDM. The present data show that in CHD and diabetic patients, in whom an activated inflammatory state is expected, inflammation as determined by serum levels of fractalkine was at the control level. Even in an elevated inflammatory state caused by postprandial hyperlipemia, no significant increase in fractalkine serum levels was found in the two patient groups compared to control subjects. The results may be explained by the quiescent disease state, optimized anti-inflammatory treatment with statins, ACE inhibitors and ARBs, and the controlled environment of the CHD and IDDM rehabilitation patients.

\section{Disclosures}

The authors report no conflicts of interest in this work. The technical assistance of Ms Claudia Pilowski and Ms Susanne Koch is gratefully acknowledged.

\section{References}

1. Ross R. The pathogenesis of atherosclerosis - an update. NEngl JMed. 1986;314:488-500.

2. Libby P. Inflammation in atherosclerosis. Nature. 2002;420: 868-874.

3. Tedgui A, Mallat Z. Cytokines in atherosclerosis: pathogenic and regulatory pathways. Physiol Rev. 2006;86:515-581.

4. Loppnow H, Werdan K, Buerke M. Vascular cells contribute to atherosclerosis by cytokine- and innate-immunity-related inflammatory mechanisms. Innate Immun. 2008;14:63-87.

5. Sun J, Sukhova GK, Wolters PJ, et al. Mast cells promote atherosclerosis by releasing proinflammatory cytokines. Nat Med. 2007;13:719-724.

6. Hansson GK, Holm J, Jonasson L. Detection of activated T lymphocytes in the human atherosclerotic plaque. Am J Pathol. 1989;135: 169-175.

7. Blankenberg S, Barbaux S, Tiret L. Adhesion molecules and atherosclerosis. Atherosclerosis. 2003;170:191-203.

8. Luster AD. Chemokines-chemotactic cytokines that mediate inflammation. N Engl J Med. 1998;338:436-445.

9. Gerszten RE, Mach F, Sauty A, Rosenzweig A, Luster AD. Chemokines, leukocytes, and atherosclerosis. J Lab Clin Med. 2000;136:87-92.

10. Bazan JF, Bacon KB, Hardiman G, et al. Fractalkine: a new class of membrane-bound chemokine with $\mathrm{CX}_{3} \mathrm{C}$ motif. Nature. 1997;385: $64-44$.

11. Imai T, Hieshima $\mathrm{K}$, Haskell $\mathrm{C}$, et al. Identification and molecular characterization of fractalkine receptor CX3CR1, which mediates both leukocyte migration and adhesion. Cell. 1997;91:521-530.

12. Harrison JK, Jiang Y, Wees EA, et al. Inflammatory agents regulate in vivo expression of fractalkine in endothelial cells of the rat heart. J Leukoc Biol. 1999;66:937-944.

13. Chapman GA, Moores KE, Gohil J, et al. The role of fractalkine in the recruitment of monocytes to the endothelium. Eur J Pharmacol. 2000; 392:189-195.
14. Ludwig A, Berkhout T, Moores K, Groot P, Chapman G. Fractalkine is expressed by smooth muscle cells in response to IFN-gamma and TNF-alpha and is modulated by metalloproteinase activity. J Immunol. 2002;168:604-612.

15. Wong BW, Wong D, McManus BM. Characterization of fractalkine (CX3CL1) and CX3CR1 in human coronary arteries with native atherosclerosis, diabetes mellitus, and transplant vascular disease. Cardiovasc Pathol. 2002;11:332-338.

16. Hundhausen C, Misztela D, Berkhout TA, et al. The disintegrin-like metalloproteinase ADAM10 is involved in constitutive cleavage of CX3CL1 (fractalkine) and regulates CX3CL1-mediated cell-cell adhesion. Blood. 2003;102:1186-1195.

17. Haskell CA, Cleary MD, Charo IF. Molecular uncoupling of fractalkinemediated cell adhesion and signal transduction. Rapid flow arrest of CX3CR1-expressing cells is independent of G-protein activation. J Biol Chem. 1999;274:10053-10058.

18. Michelsen KS, Doherty TM, Shah PK, Arditi M. TLR signaling: An emerging bridge from innate immunity to atherogenesis. J Immunol. 2004;173:5901-5907.

19. Robinson LA, Nataraj C, Thomas DW, et al. A role for fractalkine and its receptor (CX3CR1) in cardiac allograft rejection. J Immunol. 2000;165:6067-6072.

20. Kim TS, Lim HK, Lee JY, et al. Changes in the levels of plasma soluble fractalkine in patients with mild cognitive impairment and Alzheimer's disease. Neurosci Lett. 2008;436:196-200.

21. Bjerkeli V, Damas JK, Fevang B, Holter JC, Aukrust P, Froland SS. Increased expression of fractalkine (CX3CL1) and its receptor, CX3CR1, in Wegener's granulomatosis--possible role in vascular inflammation. Rheumatology. 2007;46:1422-1427.

22. Damås JK, Boullier A, Waehre T, et al. Expression of fractalkine (CX3CL1) and its receptor, CX3CR1, is elevated in coronary artery disease and is reduced during statin therapy. Arterioscler Thromb Vasc Biol. 2005;25:2567-2572.

23. McDermott DH, Halcox JP, Schenke WH, et al. Association between polymorphism in the chemokine receptor CX3CR 1 and coronary vascular endothelial dysfunction and atherosclerosis. Circ Res. 2001;89:401-407.

24. Moatti D, Faure S, Fumeron F, et al. Polymorphism in the fractalkine receptor CX3CR1 as a genetic risk factor for coronary artery disease. Blood. 2001;97:1925-1928.

25. Steiner G. Diabetes and atherosclerosis: an overview. Diabetes. 1981;30(Suppl 2):1-7.

26. Basu A, Devaraj S, Jialal I. Dietary factors that promote or retard inflammation. Arterioscler Thromb Vasc Biol. 2006;26:995-1001.

27. Graves DT, Liu R, Oates TW. Diabetes-enhanced inflammation and apoptosis: impact on periodontal pathosis. Periodontol 2000. 2007; 45:128-137.

28. Ruster $\mathrm{C}$, Wolf $\mathrm{G}$. The role of chemokines and chemokine receptors in diabetic nephropathy. Front Biosci. 2008;13:944-955.

29. Alipour A, van Oostrom AJ, Izraeljan A, et al. Leukocyte activation by triglyceride-rich lipoproteins. Arterioscler Thromb Vasc Biol. 2008; 28:792-797.

30. Wanten G, van Emst-De Vries S, Naber T, Willems P. Nutritional lipid emulsions modulate cellular signaling and activation of human neutrophils. J Lipid Res. 2001;42:428-436.

31. van Oostrom AJ, Rabelink TJ, Verseyden C, et al. Activation of leukocytes by postprandial lipemia in healthy volunteers. Atherosclerosis. 2004; 177:175-182.

32. Alipour A, Elte JW, van Zaanen HC, Rietveld AP, Cabezas MC. Postprandial inflammation and endothelial dysfunction. Biochem Soc Trans. 2007;35:466-469.

33. Norata GD, Grigore L, Raselli S, et al. Post-prandial endothelial dysfunction in hypertriglyceridemic subjects: molecular mechanisms and gene expression studies. Atherosclerosis. 2007;193:321-327.

34. Bermúdez B, López S, Pacheco YM, et al. Influence of postprandial triglyceride-rich lipoproteins on lipid-mediated gene expression in smooth muscle cells of the human coronary artery. Cardiovasc Res. 2008;79:294-303. 
35. Loppnow H, Libby P. Proliferating or interleukin-1-activated human vascular smooth muscle cells secrete copious interleukin-6. J Clin Invest. 1990;85:731-738.

36. Chen L, Frister A, Wang S, et al. Interaction of vascular smooth muscle cells and monocytes by soluble factors synergistically enhances interleukin-6 and MCP-1 production. Am J Physiol Heart Circ Physiol. 2009;296:987-996.

37. Bursill CA, Channon KM, Greaves DR. The role of chemokines in atherosclerosis: recent evidence from experimental models and population genetics. Curr Opin Lipidol. 2004;15:145-149.

38. Ghilardi G, Biondi ML, Turri O, Guagnellini E, Scorza R. Internal carotid artery occlusive disease and polymorphisms of fractalkine receptor CX3CR1: a genetic risk factor. Stroke. 2004;35:1276-1279.

39. Franklin BA, Trivax JE, Vanhecke TE. New insights in preventive cardiology and cardiac rehabilitation. Curr Opin Cardiol. 2008;23: $477-486$.

40. Alipour A, Elte JW, van Zaanen HC, Rietveld AP, Castro Cabezas M. Novel aspects of postprandial lipemia in relation to atherosclerosis. Atheroscler Suppl. 2008;9:39-44.

41. Nissen SE, Tuzcu EM, Schoenhagen P, et al. Statin therapy, LDL cholesterol, C-reactive protein, and coronary artery disease. $N$ Engl J Med. 2005;352:29-38.
42. Prasad K. C-reactive protein-(CRP)-lowering agents. Cardiovasc Drug Rev. 2006;24:33-50.

43. Apostolakis S, Krambovitis E, Vlata Z, Kochiadakis GE, Baritaki S, Spandidos DA. CX3CR1 receptor is up-regulated in monocytes of coronary artery diseased patients: impact of pre-inflammatory stimuli and renin-angiotensin system modulators. Thromb Res. 2007;121: 387-395.

44. Albert MA, Danielson E, Rifai N, Ridker PM. Effect of statin therapy on $\mathrm{C}$-reactive protein levels: the pravastatin inflammation/CRP evaluation (PRINCE): a randomized trial and cohort study. JAMA. 2001;286:64-70.

45. Wu HP, Chen CH, Hsieh HC, Liu YC. Effects of insulin and glucose on cytokine production from peripheral blood mononuclear cells. Chang Gung Med J. 2008;31:253-259.

46. Okazaki M, Iwasaki Y, Jing H, et al. Insulin enhancement of cytokineinduced coagulation/inflammation-related gene transcription in hepatocytes. Endocr J. 2008;55:967-975.

47. Husberg C, Nygård S, Finsen AV, et al. Cytokine expression profiling of the myocardium reveals a role for CX3CL1 (fractalkine) in heart failure. J Mol Cell Cardiol. 2008;45:261-269.
Vascular Health and Risk Management

\section{Publish your work in this journal}

Vascular Health and Risk Management is an international, peerreviewed journal of therapeutics and risk management, focusing on concise rapid reporting of clinical studies on the processes involved in the maintenance of vascular health; the monitoring, prevention and treatment of vascular disease and its sequelae; and the involvement of

\section{Dovepress}

metabolic disorders, particularly diabetes. This journal is indexed on PubMed Central and MedLine. The manuscript management system is completely online and includes a very quick and fair peer-review system, which is all easy to use. Visit http://www.dovepress.com/ testimonials.php to read real quotes from published authors. 